\title{
Expression of the cell adhesion molecule CD146/MCAM in non-small cell lung cancer
}

\author{
Glen Kristiansen*, Yongwei Yu, Karsten Schlüns, Christine Sers, Manfred Dietel and Iver Petersen \\ Institute of Pathology, Charité University Hospital, 10117 Berlin, Germany
}

Received September 2002

Accepted November 2002

\begin{abstract}
Expression of MCAM is observed in a variety of human malignancies. We aimed to determine the rate of MCAM expression in our non-small cell lung cancer (NSCLC) collection and to clarify its correlation with clinicopathological parameters and patient survival. 85 NSCLC were analysed immunohistochemically using a monoclonal MCAM antibody (clone N1238) on an NSCLC tissue micro array. The staining was semiquantitatively scored. We found MCAM expression in 51\% of NSCLC, preferentially squamous cell carcinomas $(p=0.004)$. No other correlations to tumour size, grade, or stage were found. Univariate survival analysis showed no significant differences of MCAM positive and negative tumours. In adenocarcinomas however, MCAM positivity was significantly associated with shorter patient survival $(p=0.016)$. We conclude, that MCAM is expressed in a high proportion of NSCLC and might be predictive of shortened patient survival in adenocarcinomas of the lung.

Colour figure can be viewed on http://www.esacp.org/acp/2003/25-2/kristiansen.htm.
\end{abstract}

Keywords: Non-small cell lung cancer, MCAM, CD146, multiple tissue array, immunohistochemistry

\section{Introduction}

Lung cancer is a major cause of death from neoplastic malignancy in the western world with 169,400 new cases and 154,900 expected deaths in the United States for 2002 alone [4]. The molecular biology of non-small cell lung cancer (NSCLC), the largest lung cancer subgroup, is under intense international investigation in order to characterise new molecular marker genes which might be helpful in diagnosis or therapy. Although cell adhesion molecules are a focus of interest in malignancies, however, little is known about CD146 (Melanoma Cell Adhesion Molecule/MCAM, Mel-CAM, MUC18) in NSCLC. MCAM is a cell adhesion molecule of the immunoglobulin (Ig) superfamily sharing considerable sequence homology with CD166/ALCAM [2,3]. Physiological CD146 expression is described, e.g., in endothelial, thymic epithelial cells and dermal keratinocytes but also in a variety of malignancies including melanoma cell lines and malig-

\footnotetext{
* Corresponding author: Glen Kristiansen, Institute of Pathology, Charité University Hospital, Schumannstr. 20/21, 10117 Berlin, Germany. Tel.: +49 30 536145; Fax: +49 30 536900; E-mail: glen. kristiansen@charite.de.
}

nant melanoma, sarcomas, placental site trophoblastic tumours, chorioncarcinomas, breast and prostate cancer. The CD146 ligand is yet unidentified and the biological role of CD146 in malignancy is largely unclear: In breast cancer CD146 was found to function as a tumour suppressor gene whereas in malignant melanoma and prostate cancer CD146 expression appeared to correlate with disease progression [1,5,6,8-15,18-20].

We aimed to evaluate CD146 protein expression in our set of NSCLC patients and to investigate the association of expression determined by immunohistochemistry with clinicopathological parameters, disease stage according to UICC and patient survival.

\section{Material and methods}

\subsection{Tumour samples}

Our collective consisted of 85 patients between age 34 and 80 years (mean 62) who underwent thoracotomy for resection of NSCLC in the Department of Surgery of Charité University Hospital from 19951997 and of whom clinical follow-up data was available. No adjuvant radiotherapy or chemotherapy was 
Table 1

Study cohort - MCAM/CD146

\begin{tabular}{lccc}
\hline & MCAM negative & MCAM positive & $p$-value \\
\hline Total no. $(n=85)$ & $42[49 \%]$ & $43[51 \%]$ & \\
Adeno & $26[31 \%]$ & $12[14 \%]$ & 0.004 \\
SCC & $15[18 \%]$ & $29[34 \%]$ & \\
LCLC & $1[1 \%]$ & $2[2 \%]$ & \\
G1/G2 & $25[30 \%]$ & $20[23 \%]$ & 0.280 \\
G3 & $17[20 \%]$ & $23[27 \%]$ & \\
pT1 & $9[11 \%]$ & $6[7 \%]$ & 0.407 \\
pT2 ${ }^{2}$ & $33[38 \%]$ & $37[44 \%]$ & \\
pN0 & $26[31 \%]$ & $22[27 \%]$ & 0.509 \\
pN1+ ${ }^{3}$ & $16[19 \%]$ & $20[23 \%]$ & \\
Stage I & $25[29 \%]$ & $17[20 \%]$ & 0.386 \\
Stage II-III & $21[25 \%]$ & $22[26 \%]$ & \\
\hline
\end{tabular}

${ }^{1} \mathrm{G} 1(n=2), \mathrm{G} 2(n=43)$.

${ }^{2} \mathrm{pT} 2(n=60), \operatorname{pT} 3(n=7), \mathrm{pT} 4(n=3)$.

${ }^{3} \mathrm{pN} 1(n=15), \mathrm{pN} 2(n=20), \mathrm{pN} 3(n=1)$.

applied before surgery. Table 1 summarises the clinicopathological characteristics according to TNM criteria of the UICC [16]. The histopathological diagnosis was established in every case according to the WHO guidelines [17].

\subsection{Tissue array generation}

A multiple tissue array was constructed as previously described [7]. Briefly, suitable areas for tissue retrieval were marked on standard hematoxylin-eosin (HE) sections, punched out of the paraffin block and inserted into a recipient block using the tissue arrayer from Beecher Instruments (Woodland, USA). The punch diameter was $0.6 \mathrm{~mm}$. The lung tumour array consisted of 127 tissue samples, 89 tumours and 38 normal tissues (either lung parenchyma or peritumoural tissue). To assure homogeneous distribution of CD146, conventional paraffin sections were cut from additional 16 cases.

\subsection{Immunohistochemistry}

The tissue array block was freshly cut $(4 \mu \mathrm{m})$, sections were mounted on superfrost slides (MenzelGläser, Braunschweig, Germany) and dewaxed with xylene and gradually hydrated. Antigen retrieval was achieved by pressure cooking in $0.01 \mathrm{M}$ citrate buffer for 5 minutes. The MCAM-antibody (clone N1238, Novocastra, UK) was diluted 1:25 using a back- ground reducing dilution buffer (DAKO, Hamburg, Germany). No other blocking agents were employed. The primary antibody was incubated at room temperature for $1 \mathrm{~h}$. Detection took place by the conventional labelled-streptavidin-biotin method (LSABkit, DAKO, Hamburg, Germany) with alkaline phosphatase as the reporting enzyme according to the manufacturer's instructions. Fast-Red (Sigma-chemicals) served as chromogen, afterwards the slides were briefly counterstained with hematoxylin and aqueously mounted.

The staining was independently examined by two clinical pathologists (GK, YY) and was semiquantitatively scored as absent, weak and strong $(0,1+, 2+)$ for each case.

\subsection{Statistical analysis}

Fishers exact test which is especially suited for small sets of data was used to determine the strength of association between the investigated parameters. To compare the staining intensities with clinicopathological parameters, $2 \times 2$ contingency tables (e.g., no MCAM staining vs. weak or strong MCAM staining and G1\&G2 vs. G3) were set up and the resultant $p$-value was calculated. Cumulative survival curves were calculated according to the Kaplan-Meier method, differences in survival time were assessed with the log-rank test. $P$-values $<0.05$ were considered significant. All calculations were performed using the statistical software package SPSS, version 10 . 


\section{Results}

Colour figure can be viewed on http://www.esacp. org/acp/2003/25-2/kristiansen.htm.

\subsection{Immunohistochemistry}

Normal lung parenchyma showed a strong staining pattern of capillaries and smooth muscles, whereas pneumocytes were negative. The basal cell layer of bronchial epithelium was positive, mature secretory epithelium was negative. In tumour tissue, MCAM immunoreactivity was observed with a cytoplasmic and membraneous staining pattern. The 16 conventional whole mount sections showed a homogeneous staining of the tumours which is a necessary condition to obtain reliable data from small tissue array punches (data not shown).

During the staining process of the tissue micro array 4 cases were lost. 42 cases (49\%) exhibited no MCAM staining (Fig. 1A). Twenty-nine cases (34\%) showed a weak staining intensity, and 14 cases $(17 \%)$ had a strong staining signal as shown in Fig. $1 \mathrm{~B} \& \mathrm{C}$. Altogether, $51 \%$ of tumours showed an expression of MCAM in this study (Table 1).

\subsection{Statistical analysis}

MCAM expression was significantly higher in squamous cell carcinomas (Table $1, p=0.004)$. No correlation was found with tumour grading $(p=0.280)$, tumour size $(p=0.407)$, nodal status $(p=0.509)$ or disease stage according to UICC ( $p=0.663)$.

For survival analysis we compared Kaplan-Meier curves resulting from MCAM positive and negative tumours. For all tumours, we found no statistically significant differences of survival times: patients of MCAM negative tumours had a median survival time of 38 months (mean survival time 35 months), opposed to a median of 24 months (mean survival time 28 months) for MCAM positive $(1+, 2+)$ tumours $(p=$ $0.486, \log$ rank test).

We repeated this analysis comparing tumours with high MCAM expression (2+) to no or low MCAM expression. Again, the differences of median survival times were insignificant, with 32 (mean 35) months for tumours with high MCAM expression and 20 (mean 20) months for the countergroup ( $p=0.185)$.

Finally, we stratified the data according to histology, grading and nodal status: high MCAM expression was associated with shorter survival times in adenocarcinomas (Fig. 2, $p=0.016$ ), in tumours with good or moderate grading $(p=0.007)$ and as a trend in nodal negative tumours $(p=0.104)$.
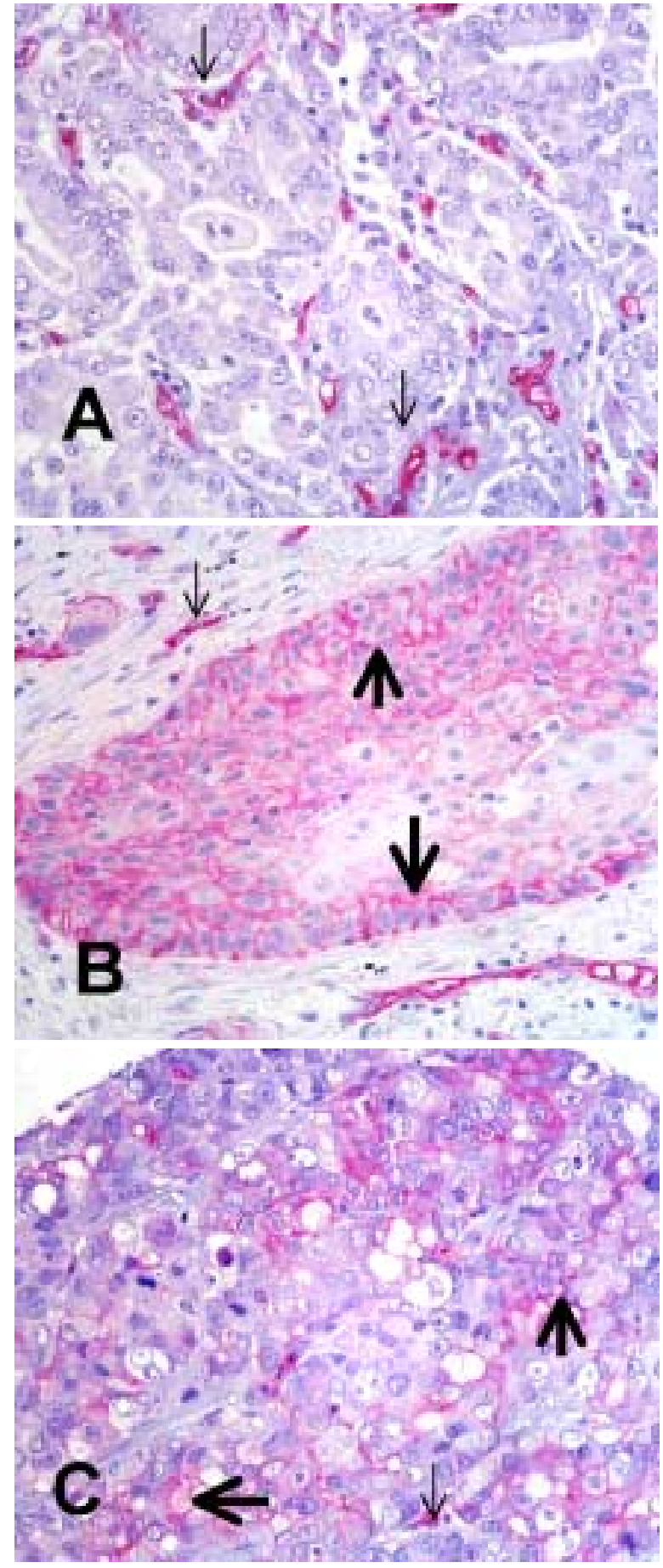

Fig. 1. MCAM immunohistochemistry in NSCLC. Thin arrows hint at the staining of capillaries. Pronounced staining of intercellular spaces is marked by thick arrows. (A) Adenocarcinoma without MCAM expression, but with a strong staining of capillaries. (B) Squamous cell carcinoma with high MCAM expression. (C) Adenocarcinoma with high MCAM expression. This figure can be viewed on http://www.esacp.org/acp/2003/25-2/kristiansen.htm. 


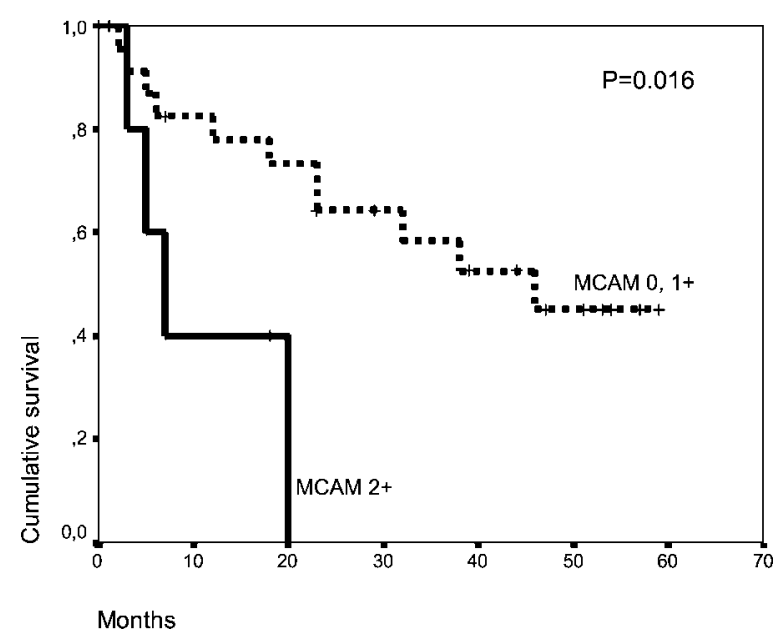

Fig. 2. Survival analysis - Kaplan-Meier curve. Adenocarcinomas only: strong MCAM expression vs. low/no MCAM expression.

\section{Discussion}

MCAM/CD146 is a $113 \mathrm{kDa}$ cell adhesion molecule of the immunoglobulin superfamily containing the characteristic V-V-C2-C2-C2 immunoglobulin-like subgroup structure in the extracellular domain. It is involved in heterotypic cell-cell interaction by binding to a ligand, which is yet unidentified. Upon activation, MCAM phosphorylates several cytoplasmic proteins, as $\mathrm{p} 125^{\mathrm{FAK}}$ and it also associates with $\mathrm{p} 59^{\mathrm{fyn}}$ and paxillin. Like many other cell surface proteins, MCAM is highly glycosylated, with apparently cell type specific carbohydrate moiety patterns. In addition to cell adhesion, MCAM appears to participate in cell signaling and possibly in the remodeling of the cytoskeleton and thus cell shape. Additionally, it might play a role in cell migration and proliferation $[14,15]$.

In this study we describe the expression of MCAM in the largest tumour collection of NSCLC published so far. Shih et al. examined MCAM in 18 NSCLC using a monoclonal MCAM antibody (clone MN-4). They found an expression rate of $30 \%$ (3/10) of squamous cell carcinomas and no MCAM expression in 10 adenocarcinomas. Due to their small tumour sample, no clinicopathological correlations were feasible. We confirm, that MCAM expression is significantly more frequent in squamous cell carcinomas (35\% of SCC). Additionally, we found MCAM expression in $14 \%$ of adenocarcinomas, which is a new finding. No other clinicopathological correlations could be demonstrated. In survival analysis, we found a shortened survival of strongly MCAM positive tumours, which was statistically insignificant $(p=0.185)$ for all tu- mours, but reached significance in adenocarcinomas $(p=0.016)$ and the group of well- or moderately graded tumours. This suggests, that MCAM expression might be associated with a more aggressive tumour phenotype and could thus be a novel prognostic marker gene in certain lung cancer subtypes, namely adenocarcinomas, which ought to be validated in a larger set of tumours. Unfortunately, our number of adenocarcinomas $(n=40)$ included in this study does not allow for a multivariate analysis. The prognostic impact of MCAM expression in adenocarcinomas hints at a possible role of MCAM in tumour progression or dissemination of adenocarcinomas of the lung.

MCAM expression or upregulation has been implicated in tumour progression in trophoblastic, vascular and smooth muscular neoplasms, in malignant melanoma, and prostate cancer $[6,11,19,20]$. In contrast, MCAM was found downregulated in breast cancer, which could indicate different MCAM functions in different tumour entities [13]. To our knowledge, our study is the first to indicate, that MCAM expression might also have prognostic value in certain tumours, as shown for adenocarcinomas of the lung. For the other tumour entities investigated for MCAM expression so far, sufficient survival analyses were not conducted.

In summary, we found that MCAM is expressed in $51 \%$ of NSCLC, mostly squamous cell carcinomas, but appears to be prognostic of shortened patient survival in adenocarcinomas on univariate analysis. This finding warrants further confirmatory studies.

\section{Acknowledgements}

Thanks to Britta Beyer for excellent technical skills and to A.E. Neumann and Marie-Christin Koll whose comments on the manuscript were very helpful.

\section{References}

[1] N. Bardin, F. Anfosso, J.M. Masse, E. Cramer, F. Sabatier, A. Le Bivic, J. Sampol and F. Dignat-George, Identification of CD146 as a component of the endothelial junction involved in the control of cell-cell cohesion, Blood 98 (2001), 3677-3684.

[2] M.A. Bowen, D.D. Patel, X. Li, B. Modrell, A.R. Malacko, W.C. Wang, H. Marquardt, M. Neubauer, J.M. Pesando, U. Francke, B.F. Haynes and A. Aruffo, Cloning, mapping, and characterization of activated leukocyte-cell adhesion molecule (ALCAM), a CD6 ligand, J. Exp. Med. 181 (1995), 1563-1568. 
[3] W.G. Degen, L.C. van Kempen, E.G. Gijzen, J.J. van Groningen, Y. van Kooyk, H.P. Bloemers and G.W. Swart, MEMD, a new cell adhesion molecule in metastasizing human melanoma cell lines, is identical to ALCAM (activated leukocyte cell adhesion molecule), Am. J. Pathol. 152 (1998), 805813.

[4] A. Jemal, A. Thomas, T. Murray and M. Thun, Cancer statistics, 2002, CA Cancer J. Clin. 52 (2002), 23-47.

[5] J.P. Johnson, J.M. Lehmann, B.G. Stade, U. Rothbacher, C. Sers and G. Riethmuller, Functional aspects of three molecules associated with metastasis development in human malignant melanoma, Invasion Metastasis 9 (1989), 338-350.

[6] J.P. Johnson, U. Rothbacher and C. Sers, The progression associated antigen MUC18: a unique member of the immunoglobulin supergene family, Melanoma Res. 3 (1993), 337-340.

[7] G. Kristiansen, Y. Yu, S. Petersen, O. Kaufmann, K. Schluns, M. Dietel and I. Petersen, Overexpression of c-erbB2 protein correlates with disease-stage and chromosomal gain at the cerbB2 locus in non-small cell lung cancer, Eur. J. Cancer $\mathbf{3 7}$ (2001), 1089-1095.

[8] A. Seftalioglu and L. Karakoc, Expression of CD146 adhesion molecules (MUC18 or MCAM) in the thymic microenvironment, Acta Histochem. 102 (2000), 69-83.

[9] C. Sers, K. Kirsch, U. Rothbacher, G. Riethmuller and J.P. Johnson, Genomic organization of the melanomaassociated glycoprotein MUC18: implications for the evolution of the immunoglobulin domains, Proc. Natl. Acad. Sci. USA 90 (1993), 8514-8518.

[10] C. Sers, G. Riethmuller and J.P. Johnson, MUC18, a melanoma-progression associated molecule, and its potential role in tumor vascularization and hematogenous spread, Cancer Res. 54 (1994), 5689-5694.
[11] I.M. Shih, T.L. Wang and W.H. Westra, Diagnostic and biological implications of mel-CAM expression in mesenchymal neoplasms, Clin. Cancer Res. 2 (1996), 569-575.

[12] I.M. Shih and R.J. Kurman, Expression of melanoma cell adhesion molecule in intermediate trophoblast, Lab. Invest. 75 (1996), 377-388.

[13] I.M. Shih, M.Y. Hsu, J.P. Palazzo and M. Herlyn, The cellcell adhesion receptor Mel-CAM acts as a tumor suppressor in breast carcinoma, Am. J. Pathol. 151 (1997), 745-751.

[14] I.M. Shih, M. Nesbit, M. Herlyn and R.J. Kurman, A new Mel-CAM (CD146)-specific monoclonal antibody, MN-4, on paraffin-embedded tissue, Mod. Pathol. 11 (1998), 1098-1106.

[15] I.M. Shih, The role of CD146 (Mel-CAM) in biology and pathology, J. Pathol. 189 (1999), 4-11.

[16] L.H. Sobin and C. Wittekind, TNM Classification of Malignant Tumours, 5th edn, Wiley-Liss Inc., 1997.

[17] W.D. Travis, WHO Histological Typing of Lung and Pleural Tumours, 3rd edn, WHO, Geneva, 1999.

[18] W. Weninger, M. Rendl, M. Mildner, C. Mayer, J. Ban, A. Geusau, G. Bayer, A. Tanew, O. Majdic and E. Tschachler, Keratinocytes express the CD146 (Muc18/S-endo) antigen in tissue culture and during inflammatory skin diseases, J. Invest. Dermatol. 115 (2000), 219-224.

[19] G.J. Wu, V.A. Varma, M.W. Wu, S.W. Wang, P. Qu, H. Yang, J.A. Petros, S.D. Lim and M.B. Amin, Expression of a human cell adhesion molecule, MUC18, in prostate cancer cell lines and tissues, Prostate 48 (2001), 305-315

[20] G.J. Wu, M.W. Wu, S.W. Wang, Z. Liu, P. Qu, Q. Peng, H. Yang, V.A. Varma, Q.C. Sun, J.A. Petros, S.D. Lim and M.B. Amin, Isolation and characterization of the major form of human MUC18 cDNA gene and correlation of MUC18 overexpression in prostate cancer cell lines and tissues with malignant progression, Gene 279 (2001), 17-31. 


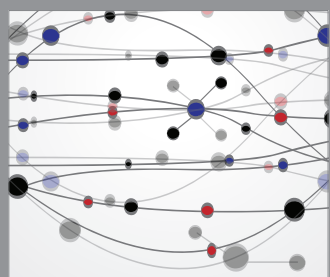

The Scientific World Journal
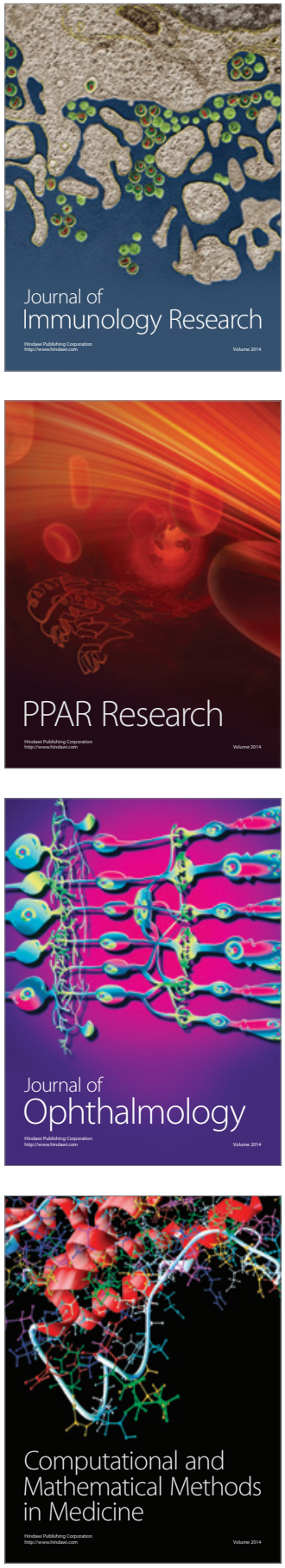

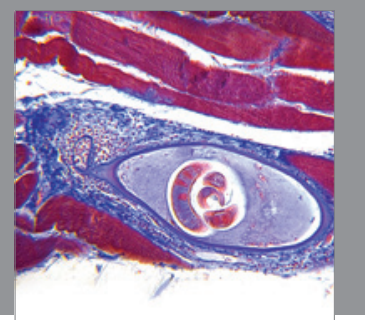

Gastroenterology

Research and Practice
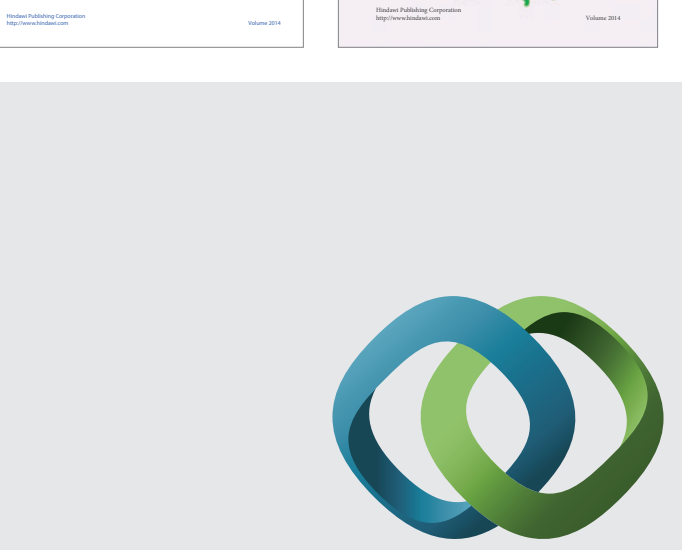

\section{Hindawi}

Submit your manuscripts at

http://www.hindawi.com
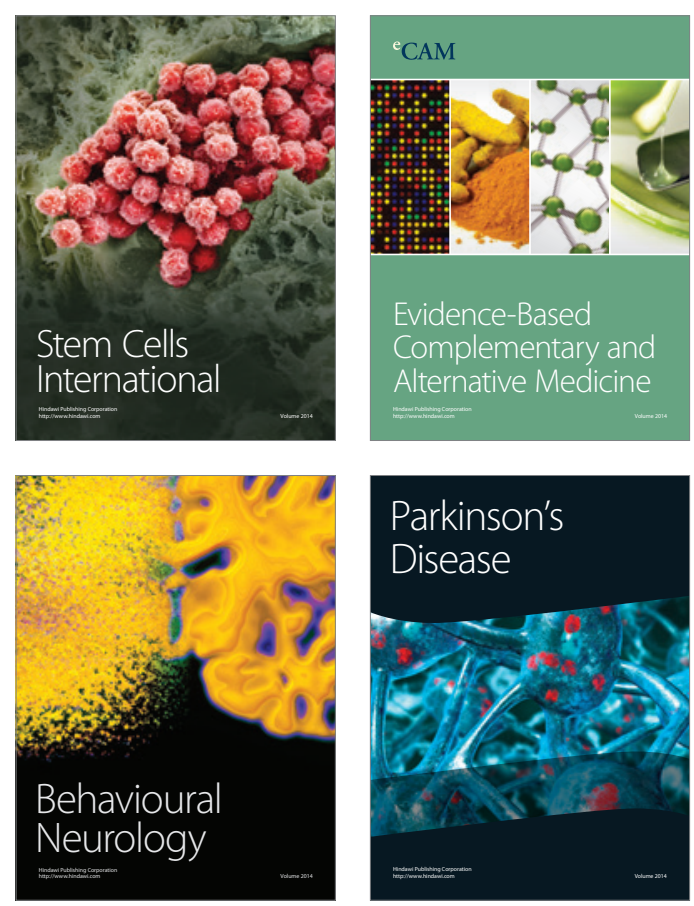

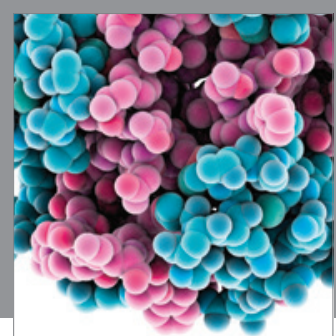

Journal of
Diabetes Research

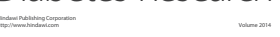

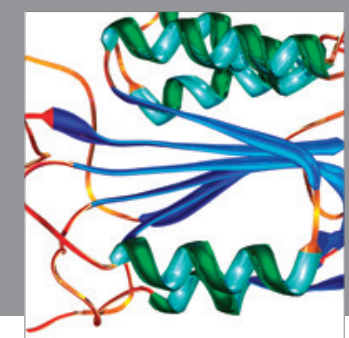

Disease Markers
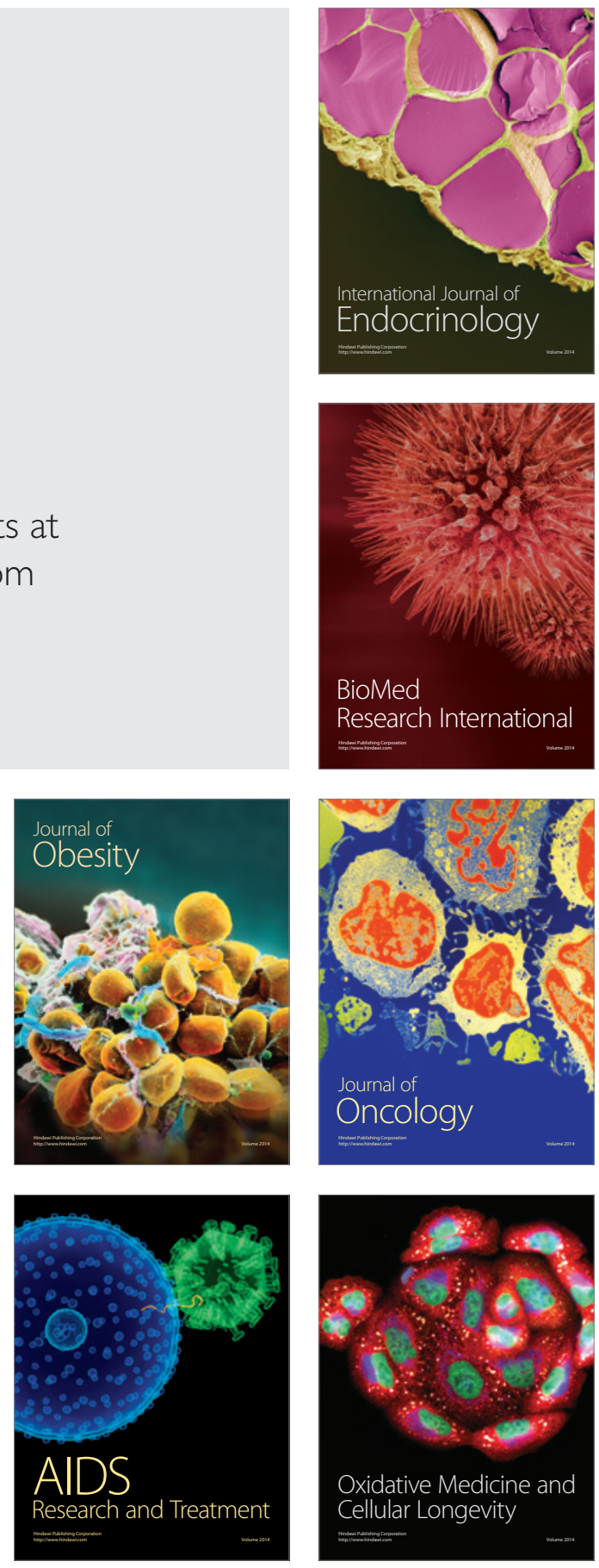